\title{
BP-SY-2-1
}

\section{Definition and diagnosis of surgical margin in extrahepatic bile duct cancer}

\author{
Haeryoung $\mathrm{KIM}^{*}$ \\ Department of Pathology, Seoul National University College of Medicine, Seoul, Korea
}

Lecture: Extrahepatic bile duct cancer is an aggressive malignant neoplasm with a dismal prognosis, and complete surgical resection of the tumor with microscopically negative (R0) margins is the only potentially curative therapy. Therefore, an accurate evaluation of the resection margin status by the pathologist is an integral part of bile duct cancer surgery, both intraoperatively (frozen section) and post-operatively (permanent section). However, intraoperative frozen section evaluation of bile duct margin status is a challenge even for experienced pathologists. In patients with bile duct cancers, ampullary cancers or pancreatic cancers with bile duct obstruction, the bile ducts proximal to the lesion are often severely inflamed due to obstruction and/or prior procedures (stent, drainage tubes etc). The bile duct mucosa of most cases demonstrate post-procedural reactive atypia, the most extreme examples being ulceration and granulation tissue formation related to metallic stent insertion. As a result, the discrimination between severe reactive atypia and high-grade dysplasia or invasive carcinoma at proximal margins is one of the most challenging tasks in frozen section pathology. Post-operative evaluation of the resected specimen may be just as challenging, and it is extremely important to ensure an accurate orientation of the specimen and to understand the surgical procedure. Whenever necessary, adequate communication with the surgeon is important, in order to fully understand the anatomical relationship and clinical context. In this talk, the pathologists' perspectives on resection margins in the context of extrahepatic bile duct cancer specimens will be discussed. 